\title{
Sperm competition and the maintenance of polymorphism
}

\author{
AG Clark ${ }^{1,2}$ \\ ${ }^{1}$ Department of Biology, Pennsylvania State University, University Park, PA 16802, USA
}

\begin{abstract}
Sperm competition may occur whenever sperm from more than one male are present in the reproductive tract of the female. Studies of field-caught Drosophila reveal that a substantial fraction $(80 \%)$ of females clearly have sperm from more than one male, and the figure is probably higher because only a small number of progeny are typically surveyed, so a strong skew in paternity can make multiplymated females appear as singly mated unless appropriate models are applied. Examination of genetic variation in aspects of sperm competition has revealed some striking patterns, particularly in the implications for the maintenance of polymorphism. The magnitude of variation in sperm competitive ability is as great as that for other fitness components, and the males with the strongest displacement also appear to be the ones with the greatest positive effect on
\end{abstract}

Keywords: sperm competition; Drosophila; polymorphism

\section{Phenomenology of sperm competition}

When a female mates with the second of two successive males before the sperm from the first male has all been used for fertilization, the sperm from the two males are in a competitive situation in which there may be differential fertilization success. Simple multiple-mating experiments with genetic markers have shown that, in Drosophila, the second male generally has the greatest paternity success, generally fathering more than $80 \%$ of the progeny (depending on the timing of the two matings and the particular lines used).

A key issue of concern for evolutionary geneticists is the variability in sperm competitive ability, because natural selection can only result in evolutionary change if there is underlying genetic variation (Birkhead, 1996). In 1995 we published a survey of genetic differences among 152 extracted chromosome lines in aspects of male mating success (Clark et al, 1995), including the ability of each line to displace sperm in females previously mated to a cn bw standard male, and the ability of the sperm from males of each line to resist being displaced by that of $\mathrm{cn}$ $b w$ males. These two aspects of sperm competition, called 'offense' and 'defense', exhibited a remarkable level of variation among lines, from those that appeared to show

Correspondence: AG Clark, Department of Biology, Pennsylvania State University, University Park, PA 16802, USA.E-mail: c92@psu.edu ${ }^{2}$ Address after 1 July 2002: Department of Molecular Biology and Genetics, Cornell University, Ithaca, NY 14853, USA fertility. Why then does not the most competitive allele simply go to fixation? Such synergistic pleiotropy makes the polymorphism even more unexpected. Examination of patterns of competitive success of pairs of male genotypes, and of female-male interactions, demonstrate clearly that the outcome of sperm competition is not a simple property of each male. That is, sperm competitive ability of male genotypes cannot simply be ranked from best to worst. Rather, the outcome of each competitive bout depends on the particular pair of males. These results have intriguing implications for the molecular biology of genes involved in the determination of sperm competitive success, and on the opportunity for maintenance of polymorphism in those genes.

Heredity (2002) 88, 148-153. DOI: 10.1038/sj/hdy/6800019

essentially no displacing ability, to those that were successful in apparently totally removing or incapacitating all sperm from the previous male. (Evidence for this is that, for some lines, in every replication, after the males from these lines were used for the second mating, every single offspring from the females was sired by the second male.) The variation among lines was highly significant, and subsequent studies established that the assays were repeatable by different investigators working in different laboratories.

At the same time that paternity success was measured, it was also possible to score the fecundity of the female, and the prior and successive mating history of the female. We had a clear expectation that there might be antagonistic pleiotropy operating, such that the alleles that confer the strongest sperm competition fare much worse in other fitness components. In particular, the observation of a strong 'cost' of mating (Chapman et al, 1995; Civetta and Clark, 2000), whereby male seminal fluids appear to have an almost toxic effect on females, suggested that perhaps the stronger displacers might be more toxic. However, we found that males that have a greater displacing ability also had a significant tendency to induce higher fecundity in a female, and a lower chance that she will remate. These pleiotropic effects represent synergistic effects with respect to fitness, and only serve to make the polymorphism for such extreme differences in fitness more unexpected. From the poorest performing male genotype to the best, the net fitnesses differed by greater than a factor of 10 . If pleiotropic effects are syner- 
gistic instead of antagonistic, it appears that we need to look elsewhere for an explanation of what could retard fixation at the loci that mediate these fitness differences.

\section{Females play an active role in sperm use}

At the outset it seems unlikely that the results of sperm competition would be determined exclusively by variation among males. Females from different genetic lines may vary in reproductive morphology, in sperm storage organs, or in biochemical response to accessory gland proteins. Therefore, it is important to determine whether the degree of sperm competition depends on the genotype of the female (Clark and Begun, 1998). Competition of sperm from two tester male genotypes $\left(b w^{D}\right.$ and B309, a third-chromosome isogenic line from Maryland, USA) was quantified by doubly mating females from 117 lines that had been rendered homozygous for $X$, second, or third chromosomes extracted from natural populations. P2, the proportion of offspring sired by the second male, was highly heterogeneous among lines, whether or not the sperm competition scores were compensated by viability estimates (Gilchrist and Partridge, 1997). These results imply that populations harbor polymorphic genes affecting the tendency of females to store the first versus the second male's sperm.

There is precedent for observing genetic variation in sperm storage among females (Yanders, 1963; DeVries, 1964), and, because there are glandular cells that secrete products into the lumen of the spermathecae (Filosi and Perotti, 1975), there is a potential cellular/molecular basis for the variation. Genetic mosaics have been used to show that the response to seminal proteins requires a female nervous system (Arthur et al, 1998). The results beg the question of molecular mechanisms that may cause these differences. Given that there appears to be chemical signaling between male and female mediated by seminal proteins, the challenge is to identify the key components of the pathway, determine how they interact, and then to return to the evolutionary genetics. One approach is to examine interspecific divergence in genes expressed in the female reproductive tract. In mammals, at least some of these genes exhibit a pattern of rapid evolution (Swanson et al, 2001a), and efforts are underway to uncover rapidly evolving female-specific genes in Drosophila as well (W Swanson, personal communication). Meanwhile, there is still more to be learned at the level of the whole-fly phenomenology of sperm competition.

\section{Male-female interaction}

Since there is clearly variation in both males and females that influences sperm competitive outcomes, the obvious next question is whether the male and female effects act independently, or whether there is some kind of interaction. An interaction would imply that male A may outperform male $\mathrm{B}$ when doubly mating with female $\mathrm{C}$, but the relative performance of the two males may be reversed with females of genotype D. Pairwise tests among six isogenic lines of Drosophila melanogaster were performed to determine whether there is a genotypespecific interaction in the success of sperm (Clark et al, 1999). Among these six lines, the success of a particular male's sperm was found to depend on the genotype of the female with which he mates (Figure 1), providing evidence for an interaction with profound evolutionary consequences. The male $\times$ female interaction in sperm precedence suggests that the proportions of offspring sired by the two males, $P 1$ and $P 2$, should be viewed as functions of the genotypes of both males and females. Population genetic models of this situation have not been formally analyzed. Parameterization would require quantities that describe the relative paternity success of male genotype $i$ when mating a female with genotype $k$ after she had already mated with male genotype $j$. With 10 genotypes, this would take 1000 parameters! Numerical simulations of a model incorporating male $\times$ female interaction with just three genotypes show that allele frequencies can exhibit quite complex behavior, including stable limit cycles. In general the time to fixation is also increased, and in this sense the complex dynamics appear to protect the polymorphism.

\section{Male-male interaction}

The simplest model for sperm competition is that there is a volumetric flushing out of resident sperm by a subsequent insemination. This model entails no specific interaction between the sperm from the two males. There is now clear experimental evidence that this model is not accurate. In Clark et al (2000), we explored two additional questions about the unit of selection that are relevant to sperm competition. First, we established that sperm competitive ability is not a property of the sperm haplotype, but rather of the diploid male's genotype. This was demonstrated by using markers such that heterozygous males produced two different phenotypes of offspring, and both differed from the phenotype of testor male offspring. Segregation of offspring genotypes from the heterozygous male was found to be the same whether the heterozygous male was the only one to mate, or was the second of two males. Then we tested whether the relative

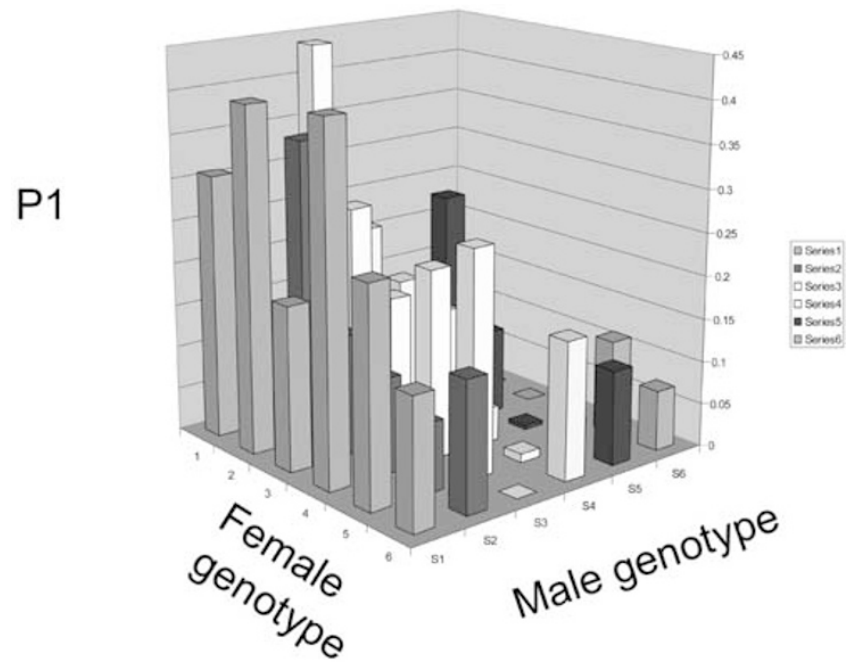

Figure 1 Sperm 'defense' parameter in a series of crosses among six strains of D. melanogaster. $P 1$ is the fraction of progeny sired by the first male in tests where the first male is of the specified genotype and the second male is a laboratory testor stock. The figure shows mean displacement parameters, and the relatively small standard errors within crosses (not shown) result in a highly significant male $\times$ female interaction (Clark et al, 1999). 
sperm competitive ability of males can be ranked on a linear array, or whether competitive ability instead depends on particular pairwise contests among males. Sperm precedence of six chromosome-extracted lines was tested against three different visible marker lines ( $c n b w$, $b w^{D}$ and $C y$ ), and the rank order of the six lines differed markedly among the mutant lines (Figure 2). Intuitively, one can see that the relative success of a particular genotype will depend on the presence and frequencies of other male genotypes in the population. Whether this kind of pairwise interaction is relevant to the maintenance of polymorphism is considered in the next section.

\section{Scramble competition}

Given that the sperm competitive success of a particular male genotype cannot be determined unless one also considers the particular genotypes against which it is competing, we need to add this complexity to population genetic models that attempt to quantify the dynamics of frequencies of alleles that influence sperm competitive ability. Early on it was recognized that one means to protect polymorphism at a locus that affects sperm competition would be to have a non-transitivity in sperm competitive results among the genotypes at one locus (Prout and Bundgaard, 1977). By this they meant that $A A$ $>A a, A a>a a$, and $a a>A A$, where $A A>A a$ implies that genotype $A A$ produced more offspring in sperm competition with genotype $A a$. To make things more complicated, we have seen that the order of the matings makes a big difference in the paternity success. The complete parameterization of such a multiple genotype model would be to specify for each pair of males a parameter $s_{i j}$ to quantify the fraction of the offspring fathered by genotype $j$ from females that have mated with males $i$ followed by $j$. This makes $s_{i j}$ the equivalent of the P2 score for male $j$ when the first male was genotype $i$. With $k$ distinct genotypes, the $s_{i j}$ values form a $k \times k$ matrix whose diagonal might be arbitrarily set to $1 / 2$. Assuming random mating, a female will mate with male $i$ followed by male $j$ with chance $p_{i} p_{j}$. For haploid transmission, assuming all females mate precisely twice, that no offspring are produced until after both matings, and that mating is at random, the model is:

$$
p_{i}^{\prime}=\frac{\sum_{j} p_{i} p_{j} s_{i j}}{\sum_{i} \sum_{j} p_{i} p_{j} s_{i j}}
$$

In the case of diploid transmission, the picture is more complicated, as one needs to enumerate the full mating table specifying progeny produced by each pairwise mating. While this would be tedious to do explicitly for a genetic system of any complexity, it is clear how to go about it.

For our purposes, we will adhere to the haploid model given above, simply because it illustrates all the points that we need to consider. Limiting the focus to the haploid model also illustrates the power of this mode of frequency dependence in maintaining polymorphism, because simple viability selection in the haploid case would drive to fixation whichever genotype has the highest fitness. In order to illustrate some intriguing attributes of this model, we made use of an unconventional source of data scores from the United States National Hockey League. While such scores bear no resemblance to the true biological actions of sperm competition (we think!), the mathematical attributes have a number of features in common. In particular, each pair of teams plays each other several times, and they play in the home cities of both teams. This gives improved accuracy in the assessment of fitnesses due to replication, and it also provides an opportunity to score both 'offense' and 'defense' asymmetries (defense games are at home and offense games are away). In addition, all sports fans know that

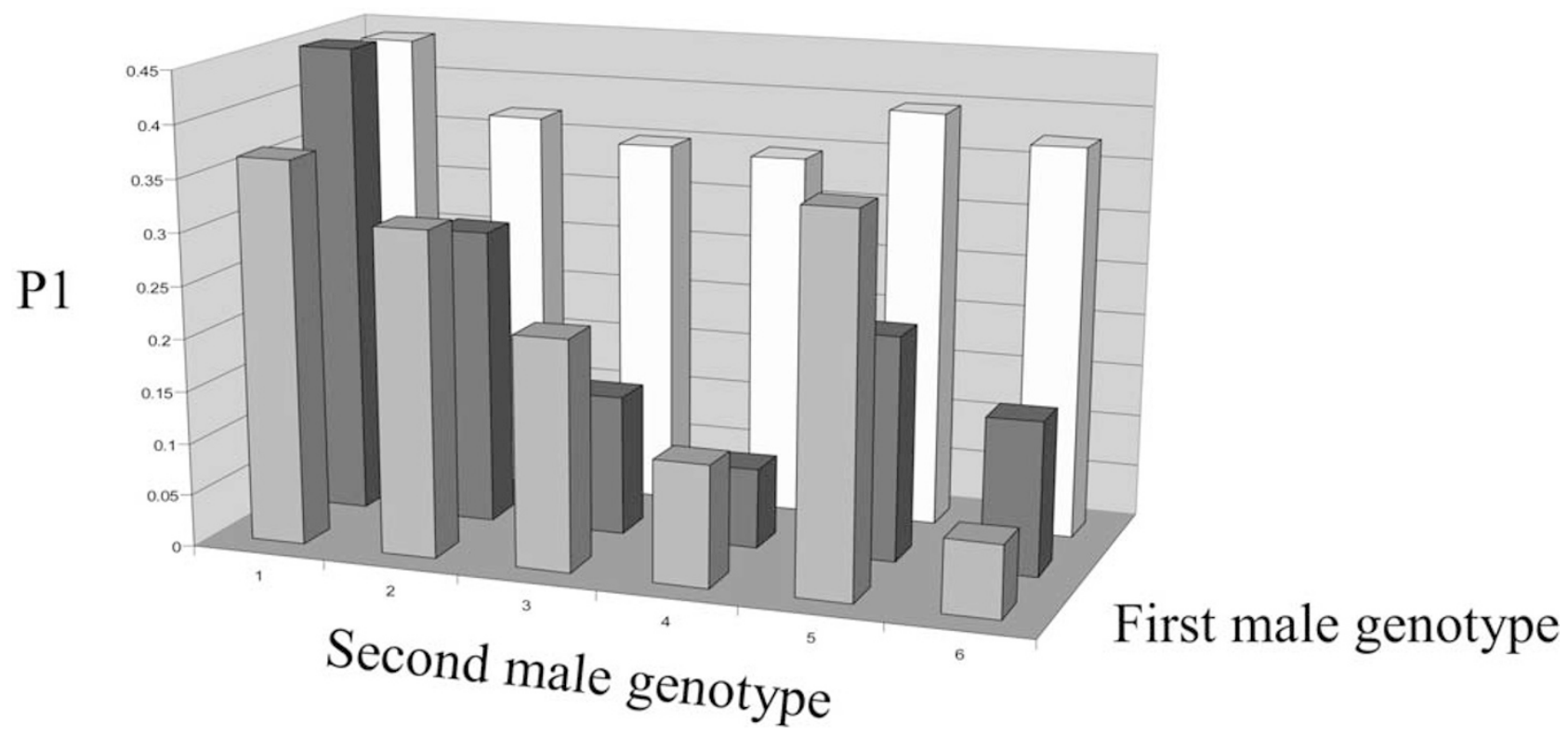

Figure 2 Sperm defense parameter in tests where the first male genotype was one of three different testor lines, and the ability of the six extracted lines to displace sperm from those testor males is compared. As in Figure 1, the line means do not show parallel trends, and there is a highly significant interaction term (Clark et al, 2000). 
an exciting season arises precisely when there is difficulty in predicting the outcome of these pairwise bouts, and that non-transitivity, or at least an unclear linear ranking of all teams, is a common occurrence. The data were retrieved from a computer database, including all 2460 games played among 30 teams in the 2001 season. We first constructed the $s_{i j}$ matrix by defining $s_{i j}$ as the fraction of points earned by team $i$ when playing team $j$ at the home location of team $i$. The recursion above was iterated from an initial condition of all teams' representation (frequency) being equal. The system came to an equilibrium with only three teams remaining, and this result was robust over many, but not all, starting conditions (Figure 3a,b).

\section{Expectations for patterns of molecular variation}

In the late 1970s there was a great deal of interest in the role of temporal and environmental heterogeneity on the maintenance of polymorphism. Initially the belief was that any such heterogeneity would serve to increase the chance for maintenance of polymorphism, simply because no single genotype could be the best in all environments. Theoretical work soon revealed that, while polymorphism could be maintained in this way, it is by no means guaranteed to maintain greater levels of genetic variation.

The model described above can retard the rate of loss of genetic variation, and in the face of mutation, this is consistent with a greater standing level of polymorphism in the underlying genes. But despite the peculiar dynamics, many alleles can go rapidly to fixation, while others can be rapidly lost. On balance, the rate of allelic turnover under this model is also expected to be accelerated. In sum, the sort of scramble competition described above is likely to produce both elevated within-species polymorphism and accelerated rates of interspecific divergence. Both of these predictions appear to be borne out by empirical examination of the patterns of molecular evolution in accessory gland proteins (Aguadé et al, 1992; Cirera and Aguadé, 1997; Civetta and Singh, 1995, 1998;
Tsaur and Wu, 1997; Tsaur et al, 1998; Aguadé, 1999a, b;

\section{Future problems}

The study of sperm competition seems to have no end to its ability to unearth new and unusual connections between biological processes. Most recently the remarkable effect of mating on immunocompetence in males (McKean and Nunney, 2001) has suggested a connection between mating and immune function. While there is no doubt that such discoveries will continue, three particular problems remain central to the evolutionary questions about sperm competition: (1) What is the association between molecular variation in Acps (accessory gland proteins) and sperm competitive success? (2) How relevant are the laboratory studies to natural conditions in the field? (3) How does the phenomenon of intraspecific sperm competition relate to conspecific precedence?

\section{Association between molecular variation and sperm competition}

There has been much progress in recent years on both the analysis of the phenomenology of genetic variation in sperm competition, and on the molecular biology of accessory gland proteins. While much evidence points to Acp variation as playing a key role in mediating sperm storage and use (Wolfner, 1997; Neubaum and Wolfner, 1999; Tram and Wolfner, 1999; and Wolfner's article in this issue), much remains to be learned about the physiological role that these proteins play. Genetic methods play a key part, and some of the clearest results have come from comparison with null mutants. It would seem that coordinated efforts in determining functional roles and the aspects of the underlying genetic variation is a particularly effective way to make progress in understanding the evolution of a phenomenon like sperm competition.

\section{b}

a
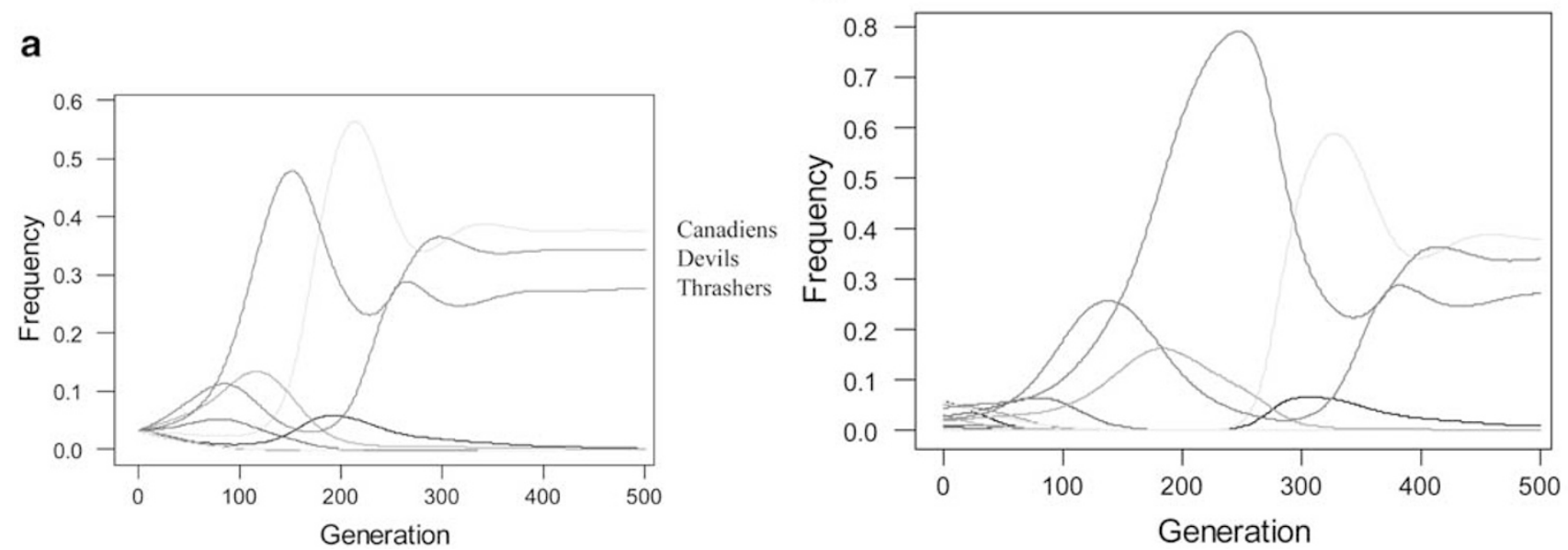

Figure 3 (a) Iterations of the haploid sperm competition model based on sperm competition parameters, $s_{i j}$, estimated from the scores of 2460 games played among 30 teams in the National Hockey League of North America. (b) provides another iteration from a slightly different initial condition. 


\section{Field studies}

In field studies of multiple mating and sperm competition typically there is no experimental control over the number of times that a female mates, the interval between matings, nor the genetic identity of multiple fathers contributing to a brood. Irrespective of this complexity, high-resolution molecular markers can be used to assign paternity with considerable confidence (Griffiths et al, 1982; Harshman and Clark, 1998; Imhof et al, 1998). Our study employed two highly heterozygous microsatellite loci to assess multiple paternity and sperm displacement in a sample of broods taken from a natural population of $D$. melanogaster. Monte Carlo simulations were used to estimate parameters for the distribution of female remating frequency and the proportion of offspring sired by the second or subsequent mating males. The mean number of males mated by a female was 1.82 , and the analog to $P 2$, estimated from doubly-mated females, was 0.79 and 0.86 for the two loci (and 0.83 for the joint estimate), so natural populations do exhibit the phenomenon of sperm precedence. The overall probability that a multiply-mated female was misclassified as singly mated was only 0.006 , indicating excellent resolution for identifying multiple mating. Studies of sperm competition in natural populations can be extended in a number of ways, including capture of flies in copula, so that at least the timing of one mating is known.

\section{Interspecific recognition}

The protein composition of seminal fluid differs widely among closely related species, and clear evidence for species specificity in accessory gland activity is obtained from transplantation and injection studies (Chen, 1996). To the extent that accessory gland secretions influence success of matings, and exhibit species-specificity, they may play an important role in the success of interspecific matings, and hence in the establishment and maintenance of mating barriers. For example, Fuyama (1983) implicated a role for accessory gland secretions in D. suzukii-D. pulchrella hybrids, and Bella et al (1992) observed strong asymmetry in homogamy among females when Chorthippus subspecies were multiply mated. Gregory and Howard (1994) and Howard et al (1998) found that the major barrier to hybridization between two related ground crickets was postmating conspecific sperm precedence. Robinson et al (1994), in double-mating studies with Tribolium castaneum and $T$. freemani, found strong conspecific sperm precedence, which they characterized as postcopulatory prezygotic isolation. Price (1997) performed tests of sperm competition among $D$. simulans, mauritiana and sechellia by mating females with conspecific males followed by heterospecific males and vice versa. In all cases there was a strong tendency for females to use conspecific sperm, as though the foreign sperm were recognized and avoided. Price et al (2000) found that when the first male to mate is conspecific, the seminal fluid from this male incapacitates subsequent sperm from heterospecific males. However, when the first mating male is heterospecific and the second male is conspecific, then the heterospecific sperm is physically removed from storage. While these phenomena look similar to what happens with all conspecific matings, it may be premature to assume that the underlying mechanism for inter- and intraspecific sperm competition are identical. The molecular mechanism for conspecific sperm recognition is of great interest, and while there is indirect evidence that Acp proteins are involved, there is no reason why the genes involved in mediating intraspecific variation in sperm competitive success are necessarily the same as those involved in mediating conspecific sperm precedence.

\section{References}

Aguadé M (1999a). Different forces drive the evolution of the Acp26Aa and Acp27Ab accessory gland genes in the Drosophila melanogaster species complex. Genetics 150: 1079-1089.

Aguadé M (1999b). Positive selection drives the evolution of the Acp29AB accessory gland protein in Drosophila. Genetics 152: 543-551.

Aguadé M, Miyashita N, Langley CH (1992). Polymorphism and divergence in the Mst26A male accessory gland gene region in Drosophila. Genetics 132: 755-770.

Arthur BI Jr, Hauschteck-Jungen E, Nothiger R, Ward PI (1998). A female nervous system is necessary for normal sperm storage in Drosophila melanogaster: a masculinized nervous system is as good as none. Proc $R$ Soc Lond B Biol Sci 265: 1749-1753.

Begun DJ, Whitley P, Todd BL, Waldrip-Dail HM, Clark AG (2000). Molecular population genetics of male accessory gland proteins in Drosophila. Genetics 156: 1879-1888.

Bella JL, Butlin RK, Ferris C, Hewitt GM (1992). Asymmetrical homogamy and unequal sex ratio from reciprocal mating order crosses between Chorthippus parallelus subspecies. Heredity 68: 345-352.

Birkhead TR (1996). Sperm competition: evolution and mechanisms. Curr Top Dev Biol 33: 103-158.

Chapman T, Liddle L, Kalb J, Wolfner M, Partridge L (1995). Cost of mating in Drosophila melanogaster females is mediated by male accessory gland products. Nature 373: 241-245.

Chen PS (1996). The accessory gland proteins in male Drosophila: structural, reproductive and evolutionary aspects. Experimentia 52: 503-510.

Cirera S, Aguadé M (1997). Evolutionary history of the sex-peptide Acp70A gene region in Drosophila melanogaster. Genetics 147: 189-197.

Civetta A, Clark AG (2000). Correlated effects of sperm competition and post-mating female mortality. Proc Natl Acad Sci USA 97: 13162-13165.

Civetta A, Singh RS (1995). High divergence of reproductive tract proteins and their association with postzygotic reproductive isolation in Drosophila melanogaster and Drosophila virilis group species. J Mol Evol 41: 1085-1095.

Civetta A, Singh RS (1998). Sex-related genes, directional sexual selection, and speciation. Mol Biol Evol 15: 901-909.

Clark AG, Aguadé M, Prout T, Harshman LG, Langley $\mathrm{CH}$ (1995). Variation in sperm displacement and its association with Accessory gland protein loci in Drosophila melanogaster. Genetics 139: 189-201.

Clark AG, Begun DJ (1998). Genetic variation in female components of sperm displacement. Genetics 149: 1487-1493.

Clark AG, Begun DJ, Prout T (1999). Female $\times$ male interactions in Drosophila sperm competition. Science 283: 217-220.

Clark AG, Dermitzakis ET, Civetta A (2000). Nontransitivity of sperm competition in Drosophila. Evolution 54: 1030-1035.

DeVries JK (1964). Insemination and sperm storage in D. melanogaster. Evolution 18: 271-282.

Filosi M, Perotti ME (1975). Fine structure of the spermatheca of Drosophila melanogaster. J Submicro Cytol 7: 259-270.

Fuyama Y (1983). Species-specificity of paragonial substances as an isolating mechanism in Drosophila. Experimentia 39: 190192.

Gilchrist AS, Partridge L (1997). Heritability of pre-adult viability differences can explain apparent heritability of sperm 
displacement ability in Drosophila melanogaster. Proc $R$ Soc Lond B Biol Sci 264: 1271-1275.

Gregory PG, Howard DJ (1994). A postinsemination barrier to fertilization isolates two closely related ground crickets. Evolution 48: 705-710.

Griffiths RC, McKechnie SW, McKenzie JA (1982). Multiple mating and sperm displacement in natural populations of Drosophila melanogaster. Theor Appl Genet 62: 89-96.

Harshman LG, Clark AG (1998). Inference of sperm competition from broods of field-caught Drosophila. Evolution 52: 13341341.

Howard D, Gregory PG, Chu J, Cain ML (1998). Conspecific sperm precedence is an effective barrier to hybridization between closely related species. Evolution 52: 511-516.

Imhof M, Harr B, Brem G, Schlötterer C (1998). Multiple mating in wild Drosophila melanogaster revisited by microsatellite analysis. Molec Ecol 7: 915-917.

McKean KA, Nunney L (2001). Increased sexual activity reduces male immune function in Drosophila melanogaster. Proc Natl Acad Sci USA 98: 7904-7909.

Neubaum DM, Wolfner MF (1999). Wise, winsome, or weird? Mechanisms of sperm storage in female animals. Curr Top Dev Biol 41: 67-97.

Price CS (1997). Conspecific sperm precedence in Drosophila. Nature 388: 663-666.

Price CS, Kim CH, Posluszny J, Coyne JA (2000). Mechanisms of conspecific sperm precedence in Drosophila. Evolution 54: 2028-2037.
Prout T, Bundgaard J (1977). Population genetics of sperm dis-

Robinson T, Johnson NA, Wade MJ (1994). Postcopulatory, prezygotic isolation: intraspecific and interspecific sperm precedence in Tribolium spp. flour beetles. Heredity 73: 155-159.

Swanson WJ, Yang Z, Wolfner MF, Aquadro CF (2001a). Positive Darwinian selection drives the evolution of several female reproductive proteins in mammals. Proc Natl Acad Sci USA 98: 2509-2514.

Swanson WJ, Clark AG, Waldrip-Dail HM, Wolfner MF, Aquadro CF (2001b). Evolutionary EST analysis identifies rapidly evolving male reproductive proteins in Drosophila. Proc Natl Acad Sci USA 98: 7375-7379.

Tram U, Wolfner MF (1999). Male seminal fluid proteins are essential for sperm storage in Drosophila melanogaster. Genetics 153: 837-844.

Tsaur S-C, Wu C-I (1997). Positive selection and the molecular evolution of a gene of male reproduction, Acp26Aa of Drosophila. Mol Biol Evol 14: 544-549.

Tsaur S-C, Ting C-T, Wu C-I (1998). Positive selection driving the evolution of a gene of male reproduction, Acp26Aa of Drosophila, II. Divergence vs. polymorphism. Mol Biol Evol 15: $1040-1046$.

Wolfner MF (1997). Tokens of love: functions and regulation of Drosophila male accessory gland products. Insect Biochem $\mathrm{Mol}$ Biol 27: 179-192.

Yanders AF (1963). The rate of Dv. melanogaster sperm migration in inter- and intraspecific matings. Dros Inf Serv 38: 33-34. 